\section{A CALL TO ACTION IN CONSULTATION TRAINING}

To the editor: We applaud the authors of "How to Give a Consultation and How to Get a Consultation" for bringing communication issues between specialists to the attention of the emergency medicine community. ${ }^{1}$ The communication skills that inform a consultation are defined by both the CanMEDS and the Accreditation Council for Graduate Medical Education (ACGME) competency frameworks. However, the educational science of a consultation is poorly studied. Hence, effective teaching strategies for both undergraduate and graduate medical education programs are potentially lacking.

A very limited number of studies have addressed consultations involving emergency physicians (EPs). The available studies focus on consultation rates, problems arising from poor communication, and disposition of patients referred to other clinical services., ${ }^{2,3}$ However, new research is addressing this literature gap.

A recent American-based study used qualitative methods to deconstruct the elements of communication between EPs and other clinical services. ${ }^{4}$ These elements inform a conceptual model termed the 5 Cs
Consultation Model: contact, communication, core question, collaboration, and closing the loop. ${ }^{4}$ Similarly, two Canadian-based abstracts, presented at the 2011 Canadian Association of Emergency Physicians (CAEP) national meeting, used qualitative methods to describe an effective consultation process from the perspectives of EPs and consultants, as well as their residents. ${ }^{5,6}$

The key global elements of a consultation from the Canadianbased research include preparation, identification of physicians and the patient, clinical questions for or from the consultants, urgency of the consultation, and feedback and followup. ${ }^{5}$ However, there were significant differences in communication priorities between residents and attending physicians as well as between clinical specialties. ${ }^{6}$

These evidence-based models may serve to standardize an effective consultation process. Our hope is that future studies will look at how these communication models can affect medical student and resident education, emergency department operations, and patient outcomes.

\section{Chad Kessler, MD, MHPE}

Section Chief of Emergency Medicine, Jesse Brown VA Medical Center, Chicago, IL

\section{Rob Woods, MD}

Emergency Physician, University of Saskatchewan, Saskatoon, SK Teresa M. Chan, BEd, MD

Resident Physician, Division of Emergency Medicine, McMaster University, Hamilton, ON

Jonathan Sherbino, MD, MEd

Division of Emergency Medicine, McMaster University, Hamilton, ON

\section{References}

1. Ackery AD, Adams JW, Brooks SC, Detsky AS. How to give a consultation and how to get a consultation. CFEM 2011;13:169-71.

2. Woods RA, Lee R, Ospina MB, et al. Consultation outcomes in the emergency department: exploring rates and complexity. C7EM 2008;10:25-31.

3. Lee R, Woods RA, Bullard M, et al. Consultations in the emergency department: a systematic review of the literature. Emerg Med 7 2008;25:4-9, doi:10.1136/emj.2007.051631.

4. Kessler CS, Kutka BM, Badillo C. Consultation in the emergency department: a qualitative analysis and review. 7 Emerg Med 2011, doi:10.1016/j .jemermed.2011.01.025.

5. Chan TM, Orlich D, Kulasegaram K, et al. Understanding communication between emergency and consulting physicians: a qualitative study that defines the essential elements of a referral-consultation [abstract]. CFEM 2011;13:178.

6. Chan TM, Orlich D, Kulasegaram K, et al. Understanding communication between emergency and consulting physicians: what are the differences between clinical specialties during the referralconsultation process? [abstract] CFEM 2011;13:207. 